\title{
RESEARCH OF THE APPLICATIONS POSSIBILITY OF INTERFACE RELAY IN HYBRID SWITCHING SYSTEMS OF BISTABLE ACTUATORS WINDINGS
}

\begin{abstract}
Purpose. Development of methods of experimental determination of action coordination characteristics of semiconductor and mechanical switching elements in systems of hybrid commutation of bistable actuators coils taking into account contact bounce of mechanical switching elements. Consideration of the application possibility of interface relay as mechanical switches, and the definition of the duration of the intervals to ensure coordination of the relay operation with semiconductor switches. Methodology. Experimental determination of the time intervals between the moments of switching transistor which controls the relay coil, and power transistor when the switching on and off operations using a hybrid switching device are performed; statistical processing of experimental results. Results. The durations of the time intervals between the moments of switching of semiconductor and mechanical switching elements in systems of actuator coils hybrid commutation are experimentally determined. A way of a significant reduction of the duration of the indicated time intervals is considered and experimentally confirmed. Originality. The scheme of the power circuit of the control system with hybrid commutation of the actuator coil circuit, which differs from the known schemes that the only semiconductor switching element performs functions of the current switching on and off, and electromechanical relay contact elements act as a router interconnecting electrical circuits during dead times. Practical value. The use of hybrid switches instead of switches with semiconductor switches will significantly reduce the cost of the actuators control system, as well as reduce their sizes. References 6 , tables 1 , figures 6 .
\end{abstract}

Key words: bistable actuators, interface relays, contact bounce, hybrid switching.

Представлены результаты экспериментальных исследований дребезга контактов интерфейсных реле при выполнении операций их включения и отключения в нормальном режиме и в режиме ускорения срабатывания. Приводятся рекомендации по применению малогабаритных интерфейсных реле в системах управления коммутационных аппаратов с гибридной коммутацией электрических цепей обмоток бистабільных актуаторов. Библ. 6, табл. 1, рис. 6. Ключевые слова: бистабильные актуаторы, интерфейсные реле, дребезг контактов, гибридная коммутация.

Introduction. Vacuum switching devices for medium voltage networks occupy a dominant position in the relevant market segment, and in the medium voltage contactors segment the share of vacuum devices is greater than $90 \%$. The invention of highly coercive permanent magnets (Masato Sagawa, 1982), as well as progress in the creation of microprocessor devices and high-power electrolytic capacitors allowed to create switching devices with highly reliable electromagnetic drives (e.g., devices by ABB circuit breakers VM1 and contactors VSC), which provide the necessary power characteristics and practicallu do not consume energy after closing and opening operations. In these devices polarized bistable actuators with two coils are used [1], and to control their winding - a microprocessor system with semiconductor switching elements - power transistor and two thyristors (Fig. 1).

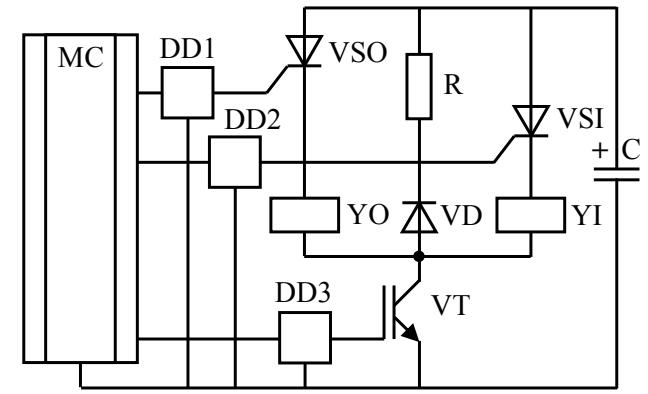

Fig. 1. Driving power circuit of activator windings control system of switching devices by ABB: YI, YO - windings on and off; VT - power transistor; VSI, VSO - thyristors, switching circuits of corresponding windings; VD - diode; $\mathrm{R}$ - resistor; DD1 ... DD3 - drivers; $\mathrm{MC}$ - microcontroller; $\mathrm{C}$ - electrolytic capacitor

In on operation, the microcontroller $\mathrm{MC}$ through driver DD3 injects the control signal to the gate of the transistor VT and through driver DD2 a short current pulse to the control electrode of the thyristor VSI, and as a result the actuator on winding YI connects to the power source, which role the role a storage electrolytic capacitor $\mathrm{C}$ performs, which is preliminary charged through the converter (not shown in the diagram) to the voltage $U_{\mathrm{C}}$. In this thyristor remains open after the termination of the control pulse. At some time, the actuator armature starts moving and after the end of its movement by the command of the position sensor the controller stops supplying the control signal to the transistor, and as a result it is locked practically immediately, and the thyristor remains in the open state, since at this moment of time the diode VD opens and current in the winding YI, closing through the thyristor VSI, diode VD and resistor $\mathrm{R}$, begins to subside by a curve close to exponential, until it drops below the level of the holding current of the thyristor VSI. At this moment, the thyristor VSI locked, simultaneously with it diode VD also locked, then the device is ready for the next operation - off operation, which is similar to on operation, but by connecting to a capacitor $\mathrm{C}$ of $\mathrm{YO}$ winding by using transistor VT and thyristor VSO.

Some disadvantage of the considered control system is the need to withstand some pause between operations that is between the moment of closing of one of thyristors (VSI or VSO) depending on the sequence of operations and transistor VT unlocking when performing a subsequent operation. It is difficult to check the fact of thyristor locking, so the algorithm of windings contrrol should assume quite a long pause (a few tens of milliseconds) between operations, else a command injection to unlock the transistor VT and thyristor VSO at

(C) B.V. Klymenko, A.V. Eres'ko, I.S. Varshamova, N.A. Lelyuk 
open thyristor VSI will not lead to the disconnection of the switching device and can cause a serious accident if it is necessary to disconnect the device immediately after switching it to a short circuit.

A polarized actuator with one coil [2] and the return spring is much simpler from designing and technological points of view, however, to perform on and off operations the coil must create oppositely MMF, which can be achieved either by winding two coils and control them alternately, either by using one coil included in the diagonal of the bridge formed by four power switching devices such as transistors. In the system shown in Fig. 2, to perform on operations (I) of the switching device, microcontroller MC through drives DD1 and DD2 injects signals to the gates of transistors VT2 and VT3, and performing the off operation $(\mathbf{O})$ - to gates of transistors VT1 and VT4.

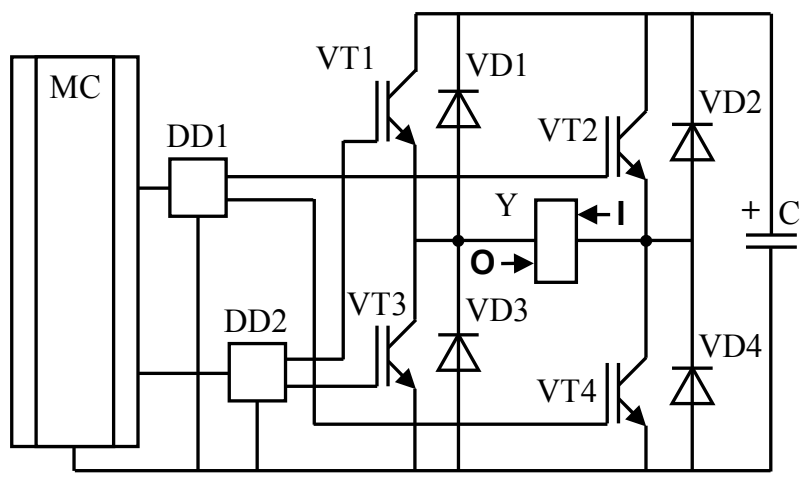

Fig. 2. Driving power circuit of actuator winding control system included in the diagonal of the bridge formed by four transistors: $\mathrm{Y}$ - actuator winding; VT1 ... VT4 - power transistors, controlled

through drivers DD1 and DD2 (by one double driver for the half-bridge); VD1 ... VD4 - diodes, providing energy recovery, accumulated in the winding, in the electrolytic capacitor $\mathrm{C}$ after locking power transistors; $\mathrm{MC}$ - microcontroller

Power transistors and drivers for them are expensive elements, therefore of interest to consider the use of a hybrid switching of actuator windings circuits. With reference to the actuator control with one winding, a power circuit may appear, for example, as shown in Fig. 3.

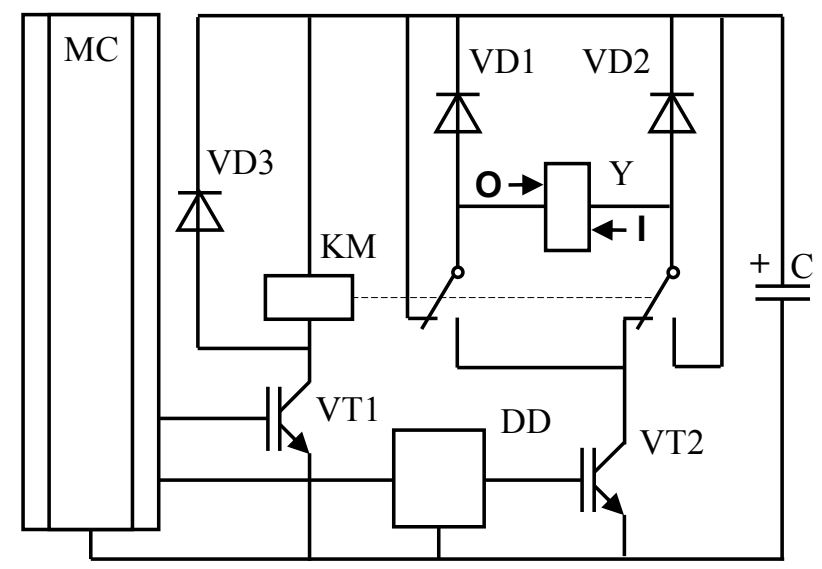

Fig. 3. Power circuit of a control system with hybrid switching of actuator winding circuit: $\mathrm{Y}$ - actuator winding; VT1 - lowpower transistor controlled directly by the microcontroller MC; VT2 - power transistor controlled through the driver DD; VD1, VD2, VD3 - diodes; C - electrolytic capacitor; KM - relay with two change-over contact groups
Hybrid switching of electrical circuits, suggesting the presence in switching devices of both mechanical (contact) and semiconductor switching elements, is one of the most promising directions of development of electric apparatus [3-6], combining advantages of circuits switching by mechanical and semiconductor switching elements. In this hybrid switching is used primarily to increase the breaking capacity and electrical durability of apparatus that perform operations on and off. In hybrid switches unlike conventional switching devices, current on and off functions are performed by only the semiconductor switching element (transistor), and the mechanical switching elements (contact groups of relays) perform the functions of a router, switching electrical circuits during dead times. The use of hybrid switches of this type instead of the existing semiconductor switches will significantly reduce the cost of the actuators control systems, as well as reduce their sizes. This direction of hybrid switching is relatively new, so the study of the characteristics of the processes that occur in such switching, have some promise both in theoretical and in practical terms.

The aim of the work. The control algorithm of relay coil and power transistor given by the microcontroller must coordinate their work clearly and avoid interruptions in the opening relay contacts at the open transistor to prevent the occurrence of the arc on the relay contacts. Any switching operation in the electromagnetic relay is accompanied by contact bounce, which is due to the peculiarities of the structure of their contact systems. Coordination of the relay and power transistor operation has to take into account and this factor - the transistor should be open not earlier, what the end of contact bounce, that is not before the operation of the relay on or off will end completely. Relay manufacturers do not present in the specifications such the characteristic as duration of a fully completed operation on and off taking into account contact bounce, accompanying an operation that does not allow the use of relay in hybrid switching systems under the manufacturers warranty. Applying one or another relay in hybrid switching system, the manufacturer of the switching device should be based on reliable results of experimental investigations, taking into account the random components of the relay timing characteristics. Here, the experiment must be based on a certain method of experimental determination of the time intervals between the moments of switching transistor which controls the relay coil, and the power transistor at performing on and off operations of the switching device, the development of which is one of the goals of this work.

Another goal is to develop recommendations for the reduction of the duration of these intervals, because their reduction improves the breaker speed - one of the most important characteristics of the switching device.

A method of experimental determination of the time intervals between the moments of switching transistor which controls the relay coil, and the power transistor. The control algorithm for transistors VT1 and VT2 in the considered system (Fig. 3) implies that for any 
switching state of the apparatus transistor VT1 initially have to be obligatory locked. This means that after the full completion of on operation of the switching device, if after this for a predetermined time interval $\Delta t$, sufficient to ensure that the current in the coil after the transistor VT2 locking decreased to near zero (this time is experimentally determined and in real apparatus does not exceed $100 \mathrm{~ms}$ ) does not come the off command, the microcontroller closes transistor VT1, with the result that the relay is switched off, its contacts come in initial condition, preparing electrical circuits to carry out the subsequent on operation. The microcontroller continuously monitors the state of the switching device, and if the ion command comes when the apparatus is switched on, no action is taken, and if the the on command comes when the apparatus is switched off, the microcontroller performs two consecutive actions: 1) sends a signal to unlock the transistor VT1, whereby, the relay $\mathrm{KM}$ switches on, the relay contacts switching state is changed, the power circuit of the control system is prepared to perform the on operation of the apparatus, and 2) sends a signal for unlocking transistor VT2, whereby the actuator is activated and the apparatus switched on. At the on operation of the apparatus, the power transistor has to be opened no earlier than the end of relay contacts bounce, so the duration of the period of time between $t_{\mathrm{bI}}$ between the moments of unlocking transistors VT1 and VT2 should be guaranteed more than the interval between the moment of voltage supply to the relay coil $\mathrm{KM}$ and the moment of guaranteed end of contact bounce.

If the external command to off the apparatus comes when the apparatus before it was switched on longer than above mentioned duration of period of time $\Delta t$, the unlocking signal to the transistor VT2 controller issues immediately. If the off command comes during the on operation switching of the switching device, the controller has to interrupt the on operation, locking the transistor VT2, wait for the signal from the apparatus contact position sensor that its main contacts come into the open state (during this time current in the actuator coil will decrease to almost zero), after that to lock transistor VT1, and to give a signal to the transistor VT2 unlocking not earlier than in the time interval $t_{\mathrm{bO}}$ between transistor VT1 locking moment and the moment of guaranteed completion of the bounce of the break contact of the relay KM.

Durations of periods of time $t_{\mathrm{bI}}$ and $t_{\mathrm{bO}}$ depend on the characteristics of the relay selected for operation in a hybrid switching system. Interface relays intended mainly for switching automation circuits have attractively small dimensions and at the same time sufficiently large current ratings surpassing the possible values of the currents in the windings of medium voltage vacuum switchgear actuators. For example, the relay RT (Schrack, Austria) or relay RM84 (ABB, Relpol, Poland) with housing dimensions of $29 \times 12.7 \times 15.7 \mathrm{~mm}$ and weight $14 \mathrm{~g}$ allow holding current in continuous mode up to $8 \mathrm{~A}$ and peak currents up to $15 \mathrm{~A}$. These relays have a sufficiently high speed - about $7 \mathrm{~ms}$ when switching on and $2 \ldots 3 \mathrm{~ms}$ when switching off. It should be borne in mind that in catalogues values of off time at mechanical opening of the relay coil circuit, which suggests that the energy stored in the winding is dissipated in the intercontact gap of the apparatus, switching this coil circuit and/or in the spark quenching circuit, connected in parallel to contacts of this device are indicated. If the switching operations are performed by a transistor interrupting the current almost immediately, the winding is required to be shunted by a «demagnetizating» diode, which opens automatically at the time of closing of the transistor shorting terminals of the winding, so that the current gradually decreases according to the law, which is close to an exponential and cumulative energy dissipates in winding resistance. As a result, the duration of fully completed operation of the switch off of the relay increases from $2 \ldots 3 \mathrm{~ms}$ (at mechanical switching off) to $16 \ldots 20 \mathrm{~ms}$. It is also important to consider the fact that there is a statistical spread of durations of periods of time $t_{\mathrm{bI}}$ and $t_{\mathrm{bo}}$ not only by comparing these characteristics at the relay from different parties, but even in the same relay. To determine $t_{\mathrm{bI}}$ и $t_{\mathrm{bO}}$ values the experiment was carried out, the scheme of which is shown in Fig. 4.

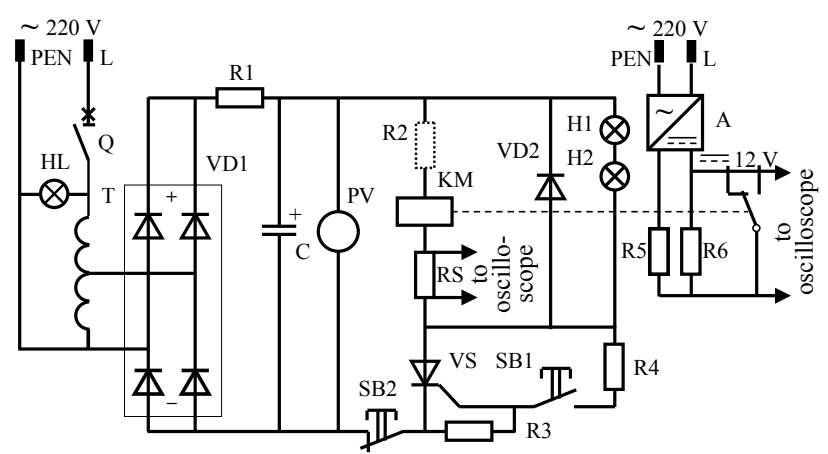

Fig. 4. The scheme of the experiment to determine the time intervals $\Delta t_{\mathrm{bI}}$ и $\Delta t_{\mathrm{bO}}: \mathrm{Q}$ - switch quaranteeing protection against overloads and short circuits; HL - warning lamp; T - autotransformer; VD1 - diode bridge; R1 - resistor, through which the capacitor $\mathrm{C}$ of high capacity (about $10,000 \mu \mathrm{F}$ ) charges; PV - voltmeter; $\mathrm{KM}$ - relay with two changeover contact groups (in the diagram one group is shown); R2 - auxiliary resistor (set if the voltage on the capacitor exceeds the nominative voltage relay coil); RS - shunt for current oscillography in the relay coil; VS - thyristor; VD2 - «demagnetizating» diode; R3, R4 resistors of the thyristor control circuit; SV1, SV2 - buttons for the relay on and off; $\mathrm{H} 1, \mathrm{H} 2$ - incandescent lamps (thyristor load); A - changer; R5, R6 - divider (registrator of the bounce of relay contacts)

A method of experimental determination of the time intervals $t_{\mathrm{bI}}$ and $t_{\mathrm{bO}}$ includes the following sequence of actions.

1. Turn the switch Q.

2. Using the autotransformer $\mathrm{T}$ set the required voltage $U_{\mathrm{C}}$ of power supply of relay (capacitor $\mathrm{C}$ ), which is controlled by the voltmeter PV.

3. Prepare the oscilloscope to the recording process.

4. Push the button SB1 and fix on the oscilloscope processes of change of current in the coil and the relay contact bounce when it is turned on. 
5. Using the autotransformer $\mathrm{T}$ set the required voltage $U_{\mathrm{C}}$ of relay power supply.

6. Prepare the oscilloscope to the recording process.

7. Push the button SB2 and fix on the oscilloscope processes of change current in the coil and the relay contact bounce when it is turned off.

Typical oscillograms of the processes on and off the relay with the power supply voltage $U_{\mathrm{C}}$ which equals to the rated supply voltage of the relay coil $U_{\mathrm{S}}$, in this case $48 \mathrm{~V}$ are shown in Fig. 5, $a$ and 5,b. From the oscillogramms it is shown that the time of relay on which is independent of the presence of «demagnetizating» diode approximatly corresponds to data of relay specification, and time off due to indivated diode is substantially greater than the value specified by the manufacturer.

Reducing the duration of intervals between the moments of switching transistor that controls the relay coil, and the power transistor. The duration of time intervals $t_{\mathrm{bI}}$ and $t_{\mathrm{bO}}$ can be significantly reduced if the relay coil with rated supply voltage $U_{\mathrm{S}}$ to connect to the source with a nominal voltage $U_{\mathrm{C}}$, superior the value $U_{\mathrm{S}}$, through a series resistor, the resistance of which is chosen so that in the steady state on the winding the established voltage equals to $U_{\mathrm{S}}$. Besides, the time constant of the relay coil circuit will increase and the current in the coil during the process of the relay on will grow faster, and in the process of the relay off will decrease rapidly until the beginning of the movement of the armature. Although, as the results of experiments show, the speed and time of armature motion as well as the time of the contacts bounce remains approximately the same as when the relay operates in the «normal» mode, the total value of the duration of the time intervals $t_{\mathrm{bI}}$ and $t_{\mathrm{bO}}$ significantly reduce compared with the corresponding durations in «normal» mode.

For example, if a circuit consisting of the relay RT winding with rated supply voltage $U_{\mathrm{S}}=48 \mathrm{~V}$, and auxiliary resistor R2, whose resistance is six times higher than winding resistance, to connect to a source voltage 7 $\times 48=336 \mathrm{~V}$, then in the steady state on the winding the established voltage will equal to $48 \mathrm{~V}$. Here, typical oscillogramms of process on and off the relay and contact bounce will appear as shown in Fig. 5,c and 5,d. From the oscillogramms it is shown that time intervals $t_{\mathrm{bI}}$ and $t_{\mathrm{bO}}$ substantially reduce compared with «normal» mode, and this being primarily due to reduction of the time constant of the winding circuit, and the slope of change of current in the winding at the end of movement of the armature as well as the duration of contacts bounce still practically the same as they were in the «normal» mode.
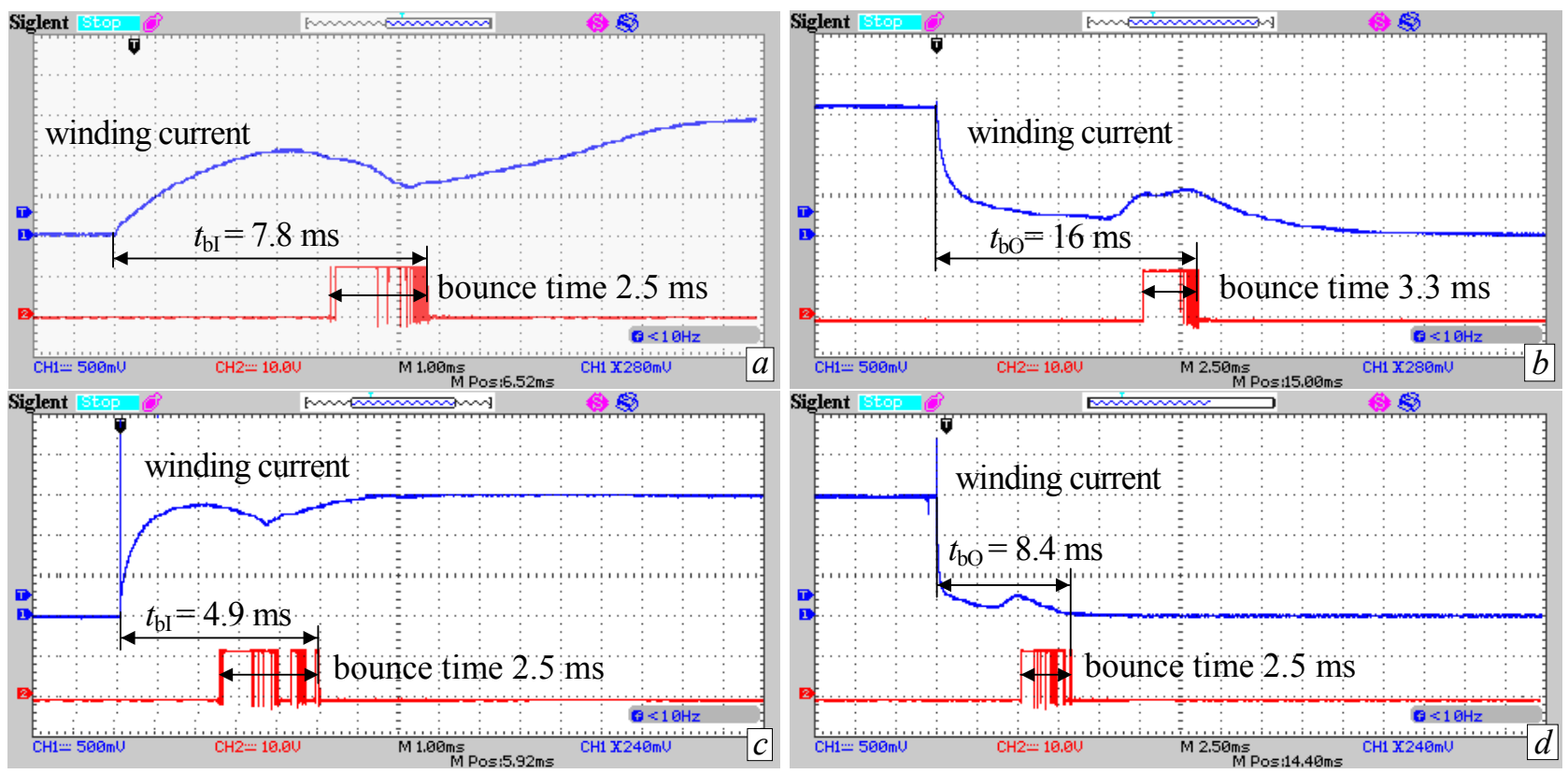

Fig. 5. Typical oscillogramms of the relay RT on and off with rated supply voltage $U_{\mathrm{S}}=48 \mathrm{~V}$ : $a$ - relay on, voltage of the control circuit (source) $U_{\mathrm{C}}=48 \mathrm{~V} ; b-$ relay off, $U_{\mathrm{C}}=48 \mathrm{~V}$; $c$ - relay on, $U_{\mathrm{C}}=336 \mathrm{~V} ; d-$ relay off, $U_{\mathrm{C}}=336 \mathrm{~V}$

The temporal characteristics of the relay of the same type (even from the same batch) may differ significantly from each other. Therefore, in order to make a correct conclusion about the necessary duration of time intervals $t_{\mathrm{bI}}$ and $t_{\mathrm{bO}}$ in hybrid switching systems, it is necessary for the selected type of relay to perform measurements of indicated durations for sufficiently large number of relays and to carry out statistical processing of experimental results. We investigated the temporal characteristics (during on and off operations) of the RT relay with winding rated supply voltage $U_{\mathrm{S}}=48 \mathrm{~V}$ at two values of the voltage of the control circuit (source ) $U_{\mathrm{C}}=48 \mathrm{~V}$ and $U_{\mathrm{C}}=336 \mathrm{~V} .20$ relays were used in the experiment with two contact groups each for a total of 40 contact groups. The experimental results are shown in Fig. 6, where by dots cumulates are shown - accumulated frequencies of values of time intervals $t_{\mathrm{bI}}$ and $t_{\mathrm{bO}}$, do not exceed the values (in $\mathrm{ms}$ ) indicated on the $x$-axis. 


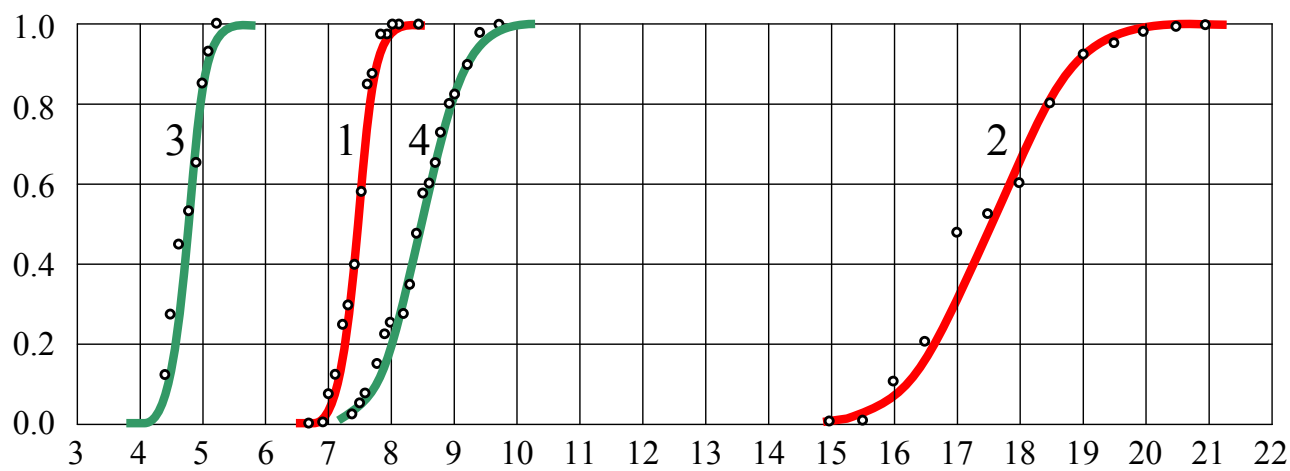

Fig. 6. Cumulates and normal distribution function, built on the results of experimental determination of the values of time intervals $t_{\mathrm{bI}}$ and $t_{\mathrm{b} \mathrm{O}}$, corresponding on and off operations of the RT relay with winding rated supply voltage $U_{\mathrm{S}}=48 \mathrm{~V}: 1-$ relay on, voltage of

the control circuit (source) $U_{\mathrm{C}}=48 \mathrm{~V} ; 2-$ relay off at $U_{\mathrm{C}}=48 \mathrm{~V} ; 3-$ relay on at $U_{\mathrm{C}}=336 \mathrm{~V} ; 4-$ relay off at $U_{\mathrm{C}}=336 \mathrm{~V}$

The solid lines in Fig. 6 show the normal distribution functions $F(t)$, built on the basis of expression

$$
F(t)=\left[1+\operatorname{erf}\left(\frac{t-\mu}{\sigma \cdot \sqrt{2}}\right)\right],
$$

where $t$ is the current value of the time interval $\left(t_{\mathrm{bI}}\right.$ or $\left.t_{\mathrm{bO}}\right)$; $\mu$ is the mathematical expectation (average value of the sample) of a random variable; $\sigma$ is the root-mean-square deviation of distribution; erf is the error function. Results of calculations of the average (according to the experimental data) values, root-mean-square values, and the probable limit values of the duration of time intervals $t_{\mathrm{bI}}$ and $t_{\mathrm{bO}}$ for the RT relay with winding rated supply voltage $U_{\mathrm{S}}=48 \mathrm{~V}$ are presented in Table. Cumulative curves in this case are very similar to the curves of normal distributions, therefore, with a high degree of reliability it can be argued that the coordination of operation of the relay RT and the semiconductor switch will be provided, if the interval between the moments of their switching will exceed the value of $\mu+3 \cdot \sigma$, taken from the last column of the Table.

Table

Results of experiment for determination of duration of time intervals $t_{\mathrm{bI}}$ and $t_{\mathrm{bO}}$ for the RT relay with winding rated supply voltage $U_{\mathrm{S}}=48 \mathrm{~V}$

\begin{tabular}{|c|c|c|c|c|}
\hline Quantity & $U_{\mathrm{C}}, \mathrm{V}$ & $\mu$ & $\sigma$ & $\mu+3 \cdot \sigma$ \\
\hline$t_{\mathrm{bI}}$ & 48 & 7.46 & 0.24 & 8.18 \\
\hline$t_{\mathrm{bO}}$ & 48 & 17.3 & 1.06 & 20.5 \\
\hline$t_{\mathrm{bI}}$ & 336 & 4.78 & 0.26 & 5.56 \\
\hline$t_{\mathrm{bO}}$ & 336 & 8.51 & 0.56 & 10.2 \\
\hline
\end{tabular}

\section{Conclusions.}

1. Switching of actuators windings circuits in which functions of the current on and off are assigned to the semiconductor switching element, and the mechanical switching elements perform the functions of routers, switching electrical circuits during the dead times, is a relatively new direction of the hybrid switching opening prospects of simplification, reduce the sizes and proce reduction of actuators control systems.

2. The hybrid switching of actuators coils circuits requires a clear coordination of moments of switching semiconductor and mechanical switching elements. The method of experimental determination of the time intervals between the moments of switching these elements taking into account contact bounce of mechanical elements is developed. The possibility of using the interface relays as mechanical switches is considered, the durations of the intervals to ensure coordination of the operation of these relays with semiconductor switches are determined.

3. A technique of a significant reduction of the duration of the intervals between the moments of switching semiconductor and mechanical switches in actuators control systems, providing the increase of speed of switching devices is considered and experimentally confirmed.

\section{REFERENCES}

1. Dullni E., Fink H., Reuber C. A vacuum circuit-breaker with permanent magnetic actuator and electronic control. Available at: https://ibrary.e.abb.com/public/5e750b2ecc5b760ec1256ad4002d2 c00/cired99 Nice VM1.pdf (accessed 15 October 2015).

2. Klymenko B.V., Vyrovets S.V., Forkun Ya.B. Elektromagnitnyi privod [Electromagnetic actuator]. Patent Russian Federation, no. 2312420, 2007. (Rus).

3. Soskov A.G., Sabalaeva N.O. Hibrydni kontaktory nyzkoi napruhy z pokrashchenymy tekhniko-ekonomichnymy kharakterystykamy: monohrafiia [Hybrid contactors low voltage with improved technical and economic characteristics]. Kharkiv, National University of Urban Economy Publ., 2012. 268 p. (Ukr).

4. Meyer J.-M., Rufer A. A DC hybrid circuit breaker with ultra-fast contact opening and integrated gate-commutated thyristors (IGCTs). IEEE Transactions on Power Delivery, 2006, vol.21, no.2, pp. 646-651. doi: 10.1109/tpwrd.2006.870981.

5. Häfner J., Jacobson B. Proactive hybrid HVDC breakers - a key innovation for reliable HVDC grids. Integrating supergrids and microgrids International Symposium in Bologna, Italy. -2011.

6. Kapoor R., Shukla A., Demetriades G. State of art of power electronics in circuit breaker technology. 2012 IEEE Energy Conversion Congress and Exposition (ECCE), 2012, pp. 615-622. doi: 10.1109/ECCE.2012.6342764.

Received 20.10.2015

B.V. Klymenko ${ }^{1}$, Doctor of Technical Science, Professor,

A.V. Eres' $\mathrm{ko}^{1}$, Candidate of Technical Science, Associate Professor,

I.S. Varshamova ${ }^{1}$, Assistant Lecturer,

N.A. Lelyuk ${ }^{1}$,

${ }^{1}$ National Technical University «Kharkiv Polytechnic Institute»,

21, Bagaleya Str., Kharkov, 61002, Ukraine.

phone: +38 050 6534982; e-mail: b.v.klymenko@gmail.com

How to cite this article:

Klymenko B.V., Eres'ko A.V., Varshamova I.S., Lelyuk N.A. Research of the applications possibility of interface relay in hybrid switching systems of bistable actuators windings. Electrical engineering \& electromechanics, 2016, no.1, pp. 21-25. doi: 10.20998/2074-272X.2016.1.04. 